\title{
Challenges and innovations of delivering medicines to older adults
}

Danijela Gnjidic ${ }^{1,2}$, Andy Husband ${ }^{3}$ and Adam Todd ${ }^{3}$

\section{Affiliations:}

1. Sydney Pharmacy School, Faculty of Medicine and Health, The University of Sydney, Sydney, NSW, Australia

2. Charles Perkins Centre, The University of Sydney, Sydney, NSW, Australia

3. School of Pharmacy, Faculty of Medical Sciences, Newcastle University, UK

\section{Corresponding author}

Dr Danijela Gnjidic

NHMRC Dementia Leadership Fellow and Senior Lecturer

Sydney School of Pharmacy, Bank Building A15, Science Rd, University of Sydney

The University of Sydney NSW 2006

Email: danijela.gnjidic@sydney.edu.au

Telephone: 61293512298 


\begin{abstract}
Older adults with multimorbidity, polypharmacy, and complex health needs are the major consumer of health care. Ensuring that medicines are used safely, effectively, and delivered efficiently in this population is challenging. In this context, the approach to medicines delivery should seek to overcome some of the difficulties of delivering medicines to older people, and ensure each medication is delivered by the optimal and most convenient route for the patient in question. However, this poses significant obstacles, as the development of medicines suitable for use in older populations does not often account for complex health needs, potential challenges in relation to drug disposition, safety of excipients and limitations with practical usability of dosage forms. The objective of this review is to summarise and discuss current challenges and novel approaches to delivering medications to older adults.
\end{abstract}

Key words: medicines, geriatric pharmacology, delivery, older people. 


\section{Introduction}

Older adults with multimorbidity, polypharmacy (multiple medicine use), functional impairment, and complex health needs are the major consumers of health care. Ensuring safe and effective medicine use in this patient group requires balance between treating multiple chronic conditions and mitigating the risks of developing medicine-related problems. According to the World Health Organisation (WHO), unsafe medication practices and medication errors are a leading cause of injury and avoidable harm in health care systems across the world, resulting in costs in excess of $\$ 42$ billion USD annually [1]. In response to this, WHO has established Medication Without Harm as the theme for the third Global Patient Safety Challenge to reduce severe avoidable medication-related harm by $50 \%$ globally in the next 5 years, with reducing polypharmacy being identified as one of the target priority areas [1]. Regardless of the approach used to measure polypharmacy among older adults, inappropriately high rates of polypharmacy have been consistently reported for several decades despite clear recognition of unsafe medication practices [2]. In Australia, polypharmacy (use of five or more medicines) is identified in $42.5 \%$ of older adults living in the community [3]. Among Americans aged 65 years and older, from 1999-2000 to 2011-2012, polypharmacy (use of five or more medicines) increased from $24.0 \%$ to $39.0 \%$ [4]. Considering the increasing trends in medication use, hyperpolypharmacy, which refers to the use of 10 or more medications, is increasingly being reported. Among older Australian adults living in the community, the prevalence of hyperpolypharmacy (use of 10 or more medicines) is $4.5 \%$, [3] compared to $23.8 \%$ among patients admitted to acute general medical hospital services [5]. Prescription medications are also increasingly used throughout Europe, with approximately $47.4 \%$ of older adults living in the community exposed to polypharmacy (use of five or more medicines), and $8.6 \%$ to hyperpolypharmacy (use of 10 or more medicines) [6].

.. Managing polypharmacy in older adults is complex: there is limited clinical evidence to support the use of complex medication regimens to manage older people with multimorbidity, while there is increasing evidence of harm, including an increased risk of an adverse event occurring, such as falling, frailty, functional impairment, hospitalization and even mortality [2]. An additional burden to ensuring the safety and efficacy of polypharmacy in older populations is ensuring appropriate delivery of medicines to older adults, and appropriate handling of medications by older adults and their caregivers. Medicines should be delivered at the correct dose and by the optimal and most convenient route of delivery for the patient in question. This process is undoubtedly more complex for older adults, as owing to multimorbidity and subsequent polypharmacy, the medication dosage 
regimens are often complex. Medication complexity is also related to number of daily administration times, drug formulations, need to split or crush tables or need to administer drugs at specific times in relation to food intake or time of day. Approaches to delivering drugs to older people can be challenging: certain medicines such as inhalers, eye drops and self-administered injections require coordination and manual dexterity, as well as other physiological characteristics, such as the ability to swallow, sufficient inspiratory flow rate and adequate vision. Ideally, formulations should be suitably designed and acceptable to patients to ensure they reflect the physiological, physical, and social challenges of ageing. Indeed, criteria to inform development of drug formulations tailored for older populations have been proposed (Figure 1) [7]; some of the key components that should be considered include bioavailability, acceptability, dose adaptation and administration, socio-cultural acceptability, and product information.



Figure 1: Criteria to inform pharmaceutical development of drug formulations. (Adapted from Breitkreut J et al 2007).

In relation to designing specific pharmaceutical products for older people, it is essential that potential handling issues, different dosing requirements and the challenges of administration issues are considered and overcome [8]. To address this, efforts around medicines development have sought to incorporate the needs of older adults when designing new pharmaceutical products [9]. This acknowledgement is significant, and it is important that this progress continues to ensure 
medicines are designed to account for the challenges of ageing to ensure they can be safely and effectively used by older people. In the sections below, we discuss the current challenges of delivering medicines in older people with respect to the natural process of ageing, multimorbidity, and patient-related factors. Finally, we consider how strategies can by employed by both a pharmaceutical industry and patient perspective to overcome these challenges to ensure medicines are delivered safely, effectively, and efficiently to older people.

\section{Age-related changes that influence drug delivery and formulation in older people}

With the normal process of ageing, there are several physiological, biological, physical and psychological changes that occur, which can have an impact on a patient's ability to achieve the best possible outcome from their medicine. The key physiological changes associated with ageing, and how such changes are likely to impact on the use and delivery of medicines in older people are briefly outlined in Table 1 [10]. With respect to pharmacokinetic changes, reduced hepatic and renal clearance are generally the most significant changes, and these are likely to affect the half-life, maintenance dose and dosing interval of a drug. The main changes in oral bioavailability with ageing result from a reduced hepatic function (reduced blood flow, and mass): the bioavailability of drugs that undergo first pass metabolism has the potential to be significantly increased. The changes in volume of distribution are smaller and affect the drug half-life and loading doses. The clinical effects of age-related pharmacodynamic changes on drug delivery are not as well established, although it is known that such changes include altered drug-receptor interactions, changes in post-receptor signalling, reduced physiological reserve and impaired homeostatic mechanics. 
Table 1: Impact of ageing on pharmacokinetics and pharmacodynamics

\begin{tabular}{|l|l|l|}
\hline Pharmacology & Physiological changes & Drug examples \\
\hline Pharmacokinetics & \begin{tabular}{l} 
Reduced gastro-intestinal surface, \\
gastric acid production, saliva \\
production \\
Reduced gastric emptying \\
\hline Ibsorption
\end{tabular} & Vitamin B12, iron \\
\hline Distribution & $\begin{array}{l}\text { Reduced renal and hepatic blood flow } \\
\text { Reduced albumin water levels } \\
\text { Reduced volume of distribution for } \\
\text { water soluble drugs } \\
\text { Increased body fat/water ratio } \\
\text { Increased volume of distribution for } \\
\text { lipid soluble drugs }\end{array}$ & $\begin{array}{l}\text { Diazepam, lignocaine, digoxin, } \\
\text { paracetamol }\end{array}$ \\
\hline Metabolism & $\begin{array}{l}\text { Reduced hepatic volume and blood } \\
\text { flow } \\
\text { Reduced hepatic mass }\end{array}$ & $\begin{array}{l}\text { Morphine, diazepam, } \\
\text { imipramine }\end{array}$ \\
\hline Excretion & $\begin{array}{l}\text { Reduced renal blood flow and } \\
\text { glomerular filtration }\end{array}$ & \begin{tabular}{l} 
Cephalexin, digoxin \\
\hline Pharmacodynamics
\end{tabular} \\
\hline & $\begin{array}{l}\text { Reduced resilience to external } \\
\text { stressors } \\
\text { Increased sensitivity to centrally active } \\
\text { drugs }\end{array}$ & $\begin{array}{l}\text { Anticholinergic drugs, sedative } \\
\text { drugs (e.g. benzodiazepines, } \\
\text { opioids), beta-blockers (e.g. } \\
\text { metoprolol) }\end{array}$ \\
\hline
\end{tabular}

In addition, in older people, many medical conditions can impair individuals' ability to take medications as intended. Medications handling and administration can be affected by chronic disease including dementia, Parkinson's disease, stroke, oesophageal disease (e.g. gastrooesophageal reflux disease), presence of visual and cognitive impairments and swallowing difficulties. Indeed, during the normal process of ageing, the swallowing reflex can change as a consequence of loss of muscle mass and reduction in the elasticity of connective tissue, thus effecting the transition of bolus down the oesophagus. For example, previous studies have identified dysphagia or impaired swallowing function in $15.0 \%$ of a general older population [11], compared to $30.0 \%$ of older people admitted to hospital [12], and $68.0 \%$ of nursing home residents [13]. A recent review reported the difficulty in swallowing oral medications among older adults ranged between $10.0 \%$ and $40.0 \%$ [14]. Evidence suggests that problems with swallowing oral dosages occur in one third of older patients which requires modification of the dosage form in terms of capsule opening and tablet crushing [15]. This may result in oral dosage forms being modified (e.g. tablets crushed or split) to facilitate administration to patients with difficulty swallowing or to tailor the dose to the patient's requirements. Studies show that $18.0 \%$ of aged care residents in Australia have received 
crushed tablets [16], compared to $32.0 \%$ of older inpatients in France [17]. Tablet splitting is also common in older adults. In a Canadian study of 370 nursing home residents $35.0 \%$ of residents received at least one split medication [18]. In addition to disease-related swallowing difficulties of medicines, other factors, such as having a previous bad experience of using a medication or disliking the idea of taking medicines, can also contribute to swallowing difficulties in older people [19]. Tablet size, shape and density, can also lead to swallowing difficulties. In addition to the physical characteristics of a tablet - such as the size, shape, and density - the colour of a tablet has also been associated with swallowing difficulties - previous studies have reported a white colour as the most popular colour choice for tablets among older adults [20].

Poor oral health may also contribute to swallowing difficulties in older people. The prevalence of xerostomia or dry mouth among community-dwelling adults ranges between $17.0 \%$ to $40.0 \%$ [21]. In older people, many medicines can cause dry mouth, which, in turn, can contribute to swallowing difficulties. Indeed, the use of benzodiazepines may affect the swallowing function secondary to the depressive action on the central nervous system [22]. Moreover, the use of antipsychotic medications in older people has been associated with oropharyngeal dysphagia [23, 24]. Interestingly, use of certain medications, such as beta-blockers, maybe associated with improved swallowing process in older people [25]. A potential mechanism of this effect is that beta-blockers reduce thickness of oral, nasal and pulmonary secretions, which may improve the swallowing function of older patients.

One approach to overcoming swallowing difficulties in older people is to use a liquid formulation; however, there are challenges in this approach, including aspiration secondary to inadequate protection of the airway during swallowing [15]. Other routes of administration, such as transdermal or parenteral drug delivery, are potentially beneficial in older people with swallowing difficulties. The transdermal route has good potential in older patients - and their caregivers - as it is simple and painless to use. The parenteral route, which is also occasionally used when an older adult has swallowing difficulties, may not be appropriate for everyone: as a result of low body weight and decreased muscle mass, frail older people can have limited venous access. Frailty, which is a multifactorial syndrome associated with functional impairment and increased susceptibility to disease, disability and mortality, can also impact on self-administration capability of medicines among older people [26]. The use of novel technologies, such as multiparticulate dosage forms, could also play a major role in delivering medicines for older adults. Multiparticulate systems, such as micropellets, offer an ideal size range for ease of administration; they also offer accurate dose 
delivery in uniform dose units, including sachet, capsules, and can be manufactured in range of dose strengths which offers important flexibility in delivering medicines to older people. Medicine lubricant can be used to mitigate swallowing difficulties. One advantage to using medicine lubricant such as Gloup available in Australia, is that it reduces the need for medicine alteration, and it may allow the patient to swallow the medicine without the need for crushing. Moreover, use of mixing vehicles such as delivering medicines with food or drink may overcome swallowing difficulties. However, it is important to consider how this may impact on drug bioavailability. For instance, use of fruit juices such as grapefruit has been shown to affect drug absorption [27]. Also, there is a need for specific information to be included in the approved product information regarding appropriate food quantity that should be used in mixing vehicles.

\section{The challenges of multimorbidity and drug delivery in an older population}

Multimorbidity, defined as the co-existence of two or more chronic conditions, is highly prevalent among older adults across the world [28]. The most common chronic conditions found in older people include: hypertension, hyperlipidaemia, ischaemic heart disease, diabetes arthritis and heart failure [29]. Overall, the number of older people with multimorbidity varies from country to country, with studies reporting prevalence rates of greater than $50.0 \%$ [30] [31], with higher prevalence reported in certain populations, such as nursing home residents (82.0\%) [32] and amongst people with intellectual disabilities (99.0\%) [33]. . By 2035, it is anticipated that the number of people living with two or more chronic conditions will increase by $86.4 \%$ - with the biggest increases observed for cancer (an increase by 179.4\%) and diabetes (an increase by 118.1\%) [34]. Why is this important in relation to delivering medicines to older people? It is has been demonstrated in the literature that the presence of multimorbidity is associated with a number of health harms, including a reduced quality of life [35], mortality [36], increased healthcare utilisation [37], and higher rates of adverse drug events [38]. In addition, in older adults, the use of polypharmacy in the context of multiple chronic conditions can be problematic owing to the potential for developing drug-disease interactions. It is possible that specific chronic conditions can influence the treatment type, dose or formulation choice for another chronic condition.

Table 2: Examples of potential drug-disease interactions occurring with NSAIDs in older people, and strategies employed to mitigate against them. 


\begin{tabular}{|c|c|c|}
\hline & using NSAIDs & overcome in older people \\
\hline Asthma & Beta-blockers & $\begin{array}{l}\text { Use another class of analgesic } \\
\text { medication; use medication } \\
\text { topically rather than systemically }\end{array}$ \\
\hline Chronic renal impairment & $\begin{array}{l}\text { Sodium and fluid retention, } \\
\text { reduced eGFR; worsening of } \\
\text { disease }\end{array}$ & $\begin{array}{l}\text { Avoid long term use of NSAIDs; } \\
\text { use medication topically rather } \\
\text { than systemically; use another } \\
\text { class of analgesic }\end{array}$ \\
\hline Congestive heart failure & $\begin{array}{l}\text { Increased fluid retention; } \\
\text { worsening of disease }\end{array}$ & $\begin{array}{l}\text { Avoid long term use of NSAIDs; } \\
\text { use medication topically rather } \\
\text { than systemically; use another } \\
\text { class of analgesic }\end{array}$ \\
\hline Hypertension & $\begin{array}{l}\text { Increased blood pressure; } \\
\text { worsening of disease }\end{array}$ & $\begin{array}{l}\text { Monitor blood pressure } \\
\text { regularly; avoid long-term use, if } \\
\text { possible }\end{array}$ \\
\hline Peptic ulcer disease (PUD) & $\begin{array}{l}\text { Gl irritation; worsening of } \\
\text { disease }\end{array}$ & $\begin{array}{l}\text { Consider using gastro-protective } \\
\text { treatment (such as a PPI or } \\
\text { H2RA) }\end{array}$ \\
\hline
\end{tabular}

Abbreviations: CNS, central nervous system; NSAIDs, non-steroidal anti-inflammatory drugs; eGFR, estimated glomerular filtration rate; GI, gastrointestinal irritation; PPI, proton pump inhibitor; H2RA, $\mathrm{H} 2$ receptor antagonist. Examples taken from [39], [40], [41].

To use non-steroidal anti-inflammatory drugs (NSAIDs) as an example - a medication commonly prescribed for older people -, these medications are associated with several clinically significant drug-disease interactions, , particularly in the presence of multimorbidity (Table 2). Worldwide, there are many examples of NSAIDs available on the market, which are widely used to treat pain and inflammation; perhaps the most notable adverse effect of the NSAIDs is gastrointestinal (GI) irritation, mediated through cyclooxygenase (COX) inhibition, which reduces the levels of GI protective prostaglandins. These Gl effects are further compounded in older people as previously discussed due to the acidic nature of NSAIDs, and the delayed gastric emptying observed in older people, as discussed in the previous section. Furthermore, as prostaglandins also dilate the afferent arterioles of the glomeruli of the kidney, the use of NSAIDs has the potential to alter kidney function - potentially leading to renal impairment; common effects of this include sodium (and thus fluid) retention, and possible hypertension [42]. In view of these well-established disadvantages, some 
treatment protocols advocate the use of other analgesics to manage pain in older people; perhaps the most common is the use of regular paracetamol, although the utility of this approach has recently been questioned [43]. If NSAIDs are indicated in older people, a simple - but effective approach to drug delivery is to reduce the potential for the systematic absorption of NSAIDs, thereby reducing the risk of adverse drug reactions and drug-disease interactions. Evidence suggests that this approach is worthwhile: a recent Cochrane review reported the efficacy of topical NSAIDs were comparable to systemic NSAIDs; that the numbers needed to treat (NNT), as defined by a $50 \%$ reduction in pain scores, for topical diclofenac and topical ketoprofen were 9.8 and 6.9 [44]. The same review also reported that, although the reporting of systemic effects of topical NSAIDs was generally of poor quality, there was no difference in observed effects between topical NSAIDs and placebo. This approach is also reflected in the UK National Institute for Health and Care Excellence (NICE) guidance when advocating the use of NSAIDs in older people: in osteoarthritis, paracetamol and topical NSAIDs are considered first line treatments; if oral NSAIDs are indicated, they should be co-prescribed with a proton pump inhibitor (PPI) to reduce the possibility of developing GI irritation [45]. Using topical NSAIDs appears to be acceptable to older people who believe topical NSAIDs are less toxic than systematic NSAIDS, and that they have a localised, rather than a generalised, effect; this is an important consideration in decision-making from both a patient, and a prescriber perspective [46].

The example of a chronic condition in an older person influencing a treatment choice or changing a specific formulation can also be extended to excipients contained within a medication. Indeed, medications can often contain high amounts of sodium or potassium, which can be problematic in some chronic diseases. For example, Gaviscon Advance ${ }^{\circledR}$ Liquid, which contains sodium alginate and potassium bicarbonate as active ingredients (specifically each $10 \mathrm{~mL}$ dose contains $4.6 \mathrm{mmol}$ sodium, and $2.0 \mathrm{mmol}$ of potassium), is often used to treat dyspepsia [47]. Due to the high sodium and potassium content contained in Gaviscon Advance ${ }^{\circledR}$, this preparation should be avoided in people with renal impairment, hypertension or heart failure - all of which are common chronic conditions in older people. Similarly, due to the problems associated with a reduced capacity to swallow in older people as discussed in the previous section, some prescribers advocate the use of effervescent formulations (e.g. soluble paracetamol tablets) as means of delivering drugs to older people. Unfortunately, this approach can also be problematic due to effervescent formulations containing high amounts of sodium chloride. For example, effervescent paracetamol formulations can contain $16.9 \mathrm{mmol}$ of sodium per $500 \mathrm{mg}$ paracetamol tablet [48], and on the basis of the WHO recommended daily intake of sodium being less than $85 \mathrm{mmol}$ per day for adults [49], a patient using 
4 grams of paracetamol daily would be taking more than the recommended daily allowance of sodium - without even considering dietary intake. In comparison to other foods that are considered high in salt, 8 effervescent paracetamol $500 \mathrm{mg}$ tablets contain nearly twice the amount of salt than $100 \mathrm{~g}$ of bacon [50]. In view of the elevated sodium levels in these products, there have been published case reports linking soluble paracetamol to hypertension [51], and hypernatraemia [52]; a further nested case controlled study using the Clinical Practice Research Datalink (CPRD) showed that exposure to sodium-containing effervescent formulations was positively - and significantly associated with increased odds of adverse cardiovascular events [53].

As well as increasing the potential for drug-disease interactions, some long-term conditions also influence the physical ability of an older person to use a medication. Examples of this include: difficulty in swallowing oral medication (e.g. people with Parkinson's disease progressively lose control of the muscles in the mouth and throat); inability to correctly use an inhaler or eye drops (e.g. people with arthritis may not have the grip strength or dexterity required to activate the inhaler); and, difficulty in removing medication from packaging (e.g. people with visual impairment may struggle to see small tablets). Given the challenges of multimorbidity in the ageing population, an older person presenting with, for example, hypertension, depression, Parkinson's disease, and osteoarthritis represents a significant challenge from a drug delivery point of view. There have been simple steps taken by the pharmaceutical industry to help overcome these challenges; these approaches include formulating oral medications as effervescent or dispersible tablets to aid swallowing; making tablets larger in size, and using colour to help with the identification and removal from packaging, and developing different inhaler types or devices that can be activated with less strength or grip. Although these attempts have been helpful, there is still much research to be done in this area to ensure patients with multimorbidity can effectively - and safely - use their medications from a physical point of review. This has been highlighted in a study by Shirmanesh and Jones who compared the physical ability of people with and without rheumatoid arthritis to use four commonly used inhaler devices [54]. The authors showed that for a pMDI, only $50.0 \%$ of rheumatoid arthritis patient could complete all of the necessary steps required in order to use the inhaler device successfully, compared to $91.0 \%$ of patients without rheumatoid arthritis; the worse performing inhaler was the HandiHaler ${ }^{\circledR}$, where only $15.0 \%$ of people with rheumatoid arthritis could complete all of the necessary steps, when compared to $94.0 \%$ of patients.

In summary, when prescribing a medication to an older person, it is important to view the patient holistically and consider any long-term conditions of the patient in any prescribing decisions, as this 
may influence the choice of medication, the dose of medication, the treatment duration, or the formulation of the medication. As well the drug therapy, the prescriber should also consider the excipients contained within a medication, and if the patient can physically use the medication as these aspects, too, could impact on the patients overall health.

\section{4. . Safe and effective packaging of medication: key challenges and considerations among older people}

In addition to drug choice and formulation considerations, the packaging of a medication is also an important factor when supplying medications to older people - this is particularly evident for older people with visual or cognitive impairment. When prescribing medication to a patient with physical of cognitive impairment, it is important for the clinician to assess the patient's capacity to use selfmanage their own medication; a number of tools have been developed to achieve this [55]. Indeed, there are many cases where harm has occurred to patients as a result of the medication being mixed up with another medication. With more and more medications being developed, there are now many examples of medications that look-a-like (Figure 3) and sound-a-like (Table 3); this refers to when names of medications have orthographic similarities and/or have similar phonetic names. It is estimated that, in the US, confusion between look-a-like or sound-a-like names contribute to approximately $25 \%$ of all medication errors [56], which, sadly, cause a number of deaths [57]. While there is an abundance of literature exploring the role of packaging in prescribing and dispensing errors [58], there is also the possibility that patients - particularly older adults with multimorbidity and polypharmacy - can mix up their medications, which can potentially cause harm. Although the packaging of the medication may not be at the front of a prescribers mind during an episode of prescribing, look-a-like or sound-a-like names should specifically be considered by the pharmacist at the point of medication supply to ensure that chances of patient harm by mixing up medication is reduced. 




Figure 3: Examples of medication packaging that 'look-a-like': atenolol $50 \mathrm{mg}$ tabs (beta-blocker) versus sertraline $50 \mathrm{mg}$ tabs (selective serotonin reuptake inhibitor) [left]; promethazine $25 \mathrm{mg} / 5 \mathrm{~mL}$ liquid (antihistamine) versus folic acid $2.5 \mathrm{mg} / 5 \mathrm{~mL}$ liquid (folate) [right].

Some possible strategies to mitigate against this is to design packaging that allows medication to stand out, such as using contrasting colours and increasing the text size on the packaging. Tallman lettering - where part of a drugs name is written in capital letters to distinguish it from other similar sounding drugs - has also been used as an approach to prevent medication errors in older people; as an example, amlodipine and atorvastatin, two commonly prescribed sound-a-like medications in older people, would be written as amLODIPine and ATORVAstatin. The use of Tallman lettering on drug packaging has been endorsed by various organisations, including the US Food and Drug Administration and, as such, is commonly used in clinical practice [59]; however, despite this widespread use, there is conflicting evidence in the literature if Tallman lettering actually prevents or reduces name confusion errors [60].

Table 3: Common medications that 'sound-a-like'.

\begin{tabular}{|l|l|l|}
\hline Medication & Sounds like & $\begin{array}{l}\text { Possible consequences of mix- } \\
\text { up }\end{array}$ \\
\hline $\begin{array}{l}\text { Aminophylline (xanthine } \\
\text { bronchodilator) }\end{array}$ & $\begin{array}{l}\text { Amitriptyline (tricyclic } \\
\text { antidepressant) }\end{array}$ & $\begin{array}{l}\text { Deterioration of asthma } \\
\text { symptoms; drowsiness; } \\
\text { increased risk of fall }\end{array}$ \\
\hline $\begin{array}{l}\text { Amiloride (potassium } \\
\text { sparing diuretic) }\end{array}$ & Amiodarone (antiarrhythmic) & $\begin{array}{l}\text { Development of cardiac } \\
\text { arrhythmias }\end{array}$ \\
\hline
\end{tabular}




\begin{tabular}{|l|l|l|}
\hline $\begin{array}{l}\text { Amlodipine (calcium } \\
\text { channel blocker) }\end{array}$ & Atorvastatin (statin) & Increase in blood pressure \\
\hline Bisoprolol (beta-blocker) & Bisacodyl (stimulant laxative) & $\begin{array}{l}\text { Increase in heart rate/elevation } \\
\text { of blood pressure; diarrhoea }\end{array}$ \\
\hline $\begin{array}{l}\text { Carbamazepine } \\
\text { (antiepileptic) }\end{array}$ & Carbimazole (antithyroid) & $\begin{array}{l}\text { Reduced seizure control; } \\
\text { reduced thyroid function }\end{array}$ \\
\hline Citalopram (SSRI) & Celecoxib (NSAID) & $\begin{array}{l}\text { Decline in mood; increase in GI } \\
\text { adverse effects }\end{array}$ \\
\hline Digoxin (cardiac glycoside) & Doxepin (SSRI) & Drowsiness; increased risk of fall \\
\hline Lofepramine (tricyclic & Loperamide (antidiarrhoeal) & Decline in mood; constipation \\
\hline $\begin{array}{l}\text { Prednisolone (a } \\
\text { corticosteroid) }\end{array}$ & Propranolol (beta-blocker) & Decline in respiratory symptoms \\
\hline Tramadol (opioid analgesic) & Trazadone (antipsychotic) & $\begin{array}{l}\text { Lack of pain control; } \\
\text { drowsiness; increase risk of fall }\end{array}$ \\
\hline
\end{tabular}

Another example of where the pharmaceutical industry has worked with medication regulators to modify drug packaging to improve the safe and effective use of medication is in Alzheimer's disease. Since 2016, in the UK, all blister packs of medication for Alzheimer's disease are marked with the days of the week in order to reduce confusion when patients take their medication [61]. While this is encouraging, given that many people with dementia also have other chronic diseases, more could be done to improve the packaging of other medicines to make them more suitable for older people with dementia.

Unfortunately there are also examples where, despite intervention from the pharmaceutical industry, the packaging and formulation of a medicine remain a problem to patient safety in older adults. Indeed, there has also been cases where older patients have mixed up different formulations of the same drug or different strengths of the same drug - which had had disastrous consequences for patients. One significant example of this is with oral methotrexate, which can be used to treat inflammatory bowel disease, rheumatoid arthritis, and psoriasis; in the UK, it is available as a $2.5 \mathrm{mg}$ tablet, and as a $10 \mathrm{mg}$ tablet. Despite varying the packaging design, the two tablet formulations could look visually similar, and when a mixture of the two strengths of methotrexate was prescribed, there were occasions where the medicines were mixed up, which lead to a number of fatalities. To mitigate against this risk, many healthcare organisations in the UK now recommend that 
methotrexate $10 \mathrm{mg}$ tablets are not prescribed for any indication, and that all doses of methotrexate are made up from $2.5 \mathrm{mg}$ tablets.

Another example is with the opioid analgesic, oxycodone; it is available as modified released tablets to be prescribed every 12 hours, and as an immediate release capsule to be prescribed every 4 to 6 hours. Despite the different formulations of oxycodone (tablets and capsules), there have been reports where older patients have mixed them up - potentially resulting in overdose of oxycodone [62]. In view of this, in a similar approach to the prescribing of methotrexate $10 \mathrm{mg}$ tablets, many healthcare organisations do not recommend the prescribing of oxycodone immediate release capsules; instead, to minimise the risk of confusion, it is recommended that oxycodone liquid is prescribed to treat pain alongside modified release oxycodone. Clearly, the decision for any healthcare organisation to not prescribe a specific medication in older adults owing to the risk in patient safety is a drastic one. While there has been advancements in recent years concerning the packaging of medication for older people, more could be done to help older people use their medication more safely and effectively. This is certainly a priority for future research.

\section{Adherence and administration: impact on drug delivery and formulation in older people}

Adherence to medicines is a modifiable factor within the process of optimising therapy and, therefore, improving it has the potential to improve patient outcomes. Patients who adhere to treatment are, on average, three times more likely to experience good health outcomes, compared to patients who are not adherent to treatment [63]. This can be challenging in older people as they often use complex medication regimens that result in a high pill burden and polypharmacy. At present, however, it is estimated that around $50 \%$ of patients do not take their medicines as prescribed by either completely missing doses [64] or taking a different dose than was prescribed. This non-adherence is more commonly associated with patient's taking less medicine, rather than taking more medicine, which has the potential to result in overdosing [65]. Occasional nonadherence is also an issue in older people, with over $85 \%$ of patients being non-adherent for some of the time and then reverting to following prescribed directions [66]. Overall, the challenges of nonadherence in older people has significant effects in terms of lost health gain and further morbidity and mortality; for example, a study by Banik and Ray suggested that more than 125,000 people in the US died as a consequence of poor adherence to prescribed medication [67]. [68]. Age is an important factor when considering adherence in older people, but studies have shown the relationship between age and adherence is complex. Rolnick et al who undertook a retrospective analysis of patients with eight different conditions measured medication possession ratios (MPR) 
and defined any patient with an MPR of $>80 \%$ as being adherent. Results of the study showed that older patients were not always less adherent and, in certain disease states, such as hypertension, hyperlipidaemia, asthma/COPD, diabetes and osteoporosis, they demonstrated marginally better adherence than younger patients [69]. In view of these findings, it would seem that age alone is not a good predictor of potential non-adherence to treatment [70]; however, there are undoubtedly challenges for older patients with multimorbidity, some of these are discussed in further detail below. A range of patient-related factors (e.g. polypharmacy, swallowing difficulties, and medication regimen complexity) and the medicine product design (e.g. different formulations) can lead to poor adherence in older adults.

\subsection{Polypharmacy and regimen complexity}

An important consideration in older adults is that polypharmacy - which is commonly observed in older people - may increase the risk of developing drug interactions and adverse effects, which, in turn, may influence a patient's decision to adhere to treatment [71]. Another important issue for many older people with complex medicine regimens is the availability of the caregiver to provide reminders to take medicines. Indeed, in a survey study by Smith et al who investigated the experiences of caregivers who manage medications for older people, one of the key themes identified was the challenges of assisting people to take their medicines and the frequency with which patients need to be reminded to physically take their treatments [72].

As a strategy to rationalise the complexity of medication regimens, the use of fixed dose combinations or 'polypills' have been shown to be beneficial. Selak et al examined the effect of a fixed dose combination of a statin, antiplatelet and two or more antihypertensive agents amongst 513 patients (average age 62 years of age) in New Zealand with high cardiovascular risk. The study demonstrated that the patients taking the fixed dose combination had better adherence compared to those using standard care; interestingly, however, despite the improved adherence, there was no statistical difference of cardiovascular risk factors between groups [73]. Systematic review of clinical outcomes associated with medication regimen complexity in older people has found mixed evidence [74]. At present, there is moderate-quality evidence that medication regimen complexity is associated with non-adherence.[75]. Other approaches that can be used by health professionals to simplify regimen complexity may include use of long-acting formulations, consolidation of dose times and avoid the need for tablet splitting. Validated tools such as the Medication Regimen Simplification Guide for Residential Aged CarE (MRS GRACE), can assist clinicians to identify 
opportunities to simplify medication regimens among older adults residing in aged care facilities [76].

\subsection{Formulation considerations}

As discussed already, the ability of an older person to swallow a dosage form or to have sufficient manual dexterity and vision to use other delivery mechanisms, such as inhalers or eye drops, has the potential to influence adherence, and thus the overall delivery of a medicine. The acceptability of a medicinal product has previously been defined in the literature as 'the overall ability of the patient and caregiver to use the medicinal product as intended or authorised' [77]. The reality of achieving this within the care of older people is influenced by a number of challenges. In the context of taking medicines, people who have swallowing difficulties are more likely to be exposed to medication errors (e.g. through unauthorised tablet crushing)[78]. [79]. ,'[80].

Moreover, the use of soluble, oral dispersible or chewable tablets is also an option for patients who have dysphagia. While this approach may improve medicine adherence in older people, there are still challenges: these formulations are not commercially available for all medicines, and for the preparations that are available, they often have a high sodium load, which, as we have discussed previously, can be problematic for older people with hypertension and cardiovascular morbidity [81] [53]. There is also some evidence to suggest that the carbon dioxide produced as a consequence of the effervescence has the potential to improve swallowing via stimulation of the oral cavity [82]. There is clearly a balance to be considered when prescribing a soluble preparation for an older person: chewable tablets are of limited use in older people, partially due to the decline in chewing ability, which is associated with loss of muscle mass and declining oral health. The products available on the market are highly variable and whilst they are all marketed as 'chewable', they all vary in terms of how easy it is for an older person to manage [83]. Oral dispersible tablets tend to be well tolerated by patients: they have been described to taste reasonably good and are potentially easier to swallow as they require less effort and avoid the need for large volumes of sodium-rich water to be consumed alongside them. [84] They have potential to help in the delivery of medicines to older people, but, unfortunately, such formulations are, at present limited. Depending on co-morbid conditions and the commercial availability of the product in question different routes may be more or less suitable depending on individual circumstances.

Inhalers 
Drugs delivered to the lungs via inhaled formulations can be challenging to use and may lead to poor medication adherence. Inhalers of all types require co-ordination in order for a drug to be delivered effectively. Older people commonly demonstrate poor inhaler technique [85] - usually as a consequence of muscle weakness in the hands, lack of co-ordination, or cognitive decline [86]. Evidence for poor inhaler technique exists for older people using all types of device, including when inhalers are combined with spacer devices [87]. Importantly, poor inhaler technique is clearly linked with worsening symptoms, the use of rescue therapy, such as oral corticosteroids, and hospital admission [87]. Although the literature has shown that older people can have poor inhaler technique with all devices, the choice of device is still an important consideration in his patient group. For example, studies have shown that adherence to treatment with inhaled corticosteroids is better for patients using dry powder inhalers (DPIs), when compared to using pressurised metered dose inhalers (pMDIs) [88]. This finding may be tempered to a degree by the issue of inspiratory effort and being able to generate sufficient flow to ensure that dry powder devices work properly. This is a concern for older patients who may have reduced peak inspiratory flow rate as a result of ageing per se, or as a consequence of comorbid disease, such as COPD [89]. Reduced inspiratory effort has the potential to limit the fraction of dose in the correct particle size range to be inhaled and deposited in the lungs. Instead, there are a greater number of larger particles produced, which are not inhaled, but deposited in the mouth and oropharynx, thus creating the potential for adverse effects, such as oral candidiasis, which, in turn, may influence the desire to adhere. The presumption, therefore, that DPIs are easier to handle, and more appropriate for older people is not always necessarily the case and, in fact, handling errors with DPIs are not lower than those associated with pMDIs [87]. The issue of supporting older patients to use inhalers more effectively is discussed further below.

\subsection{Interventions to improve medication adherence and administration}

Whilst the delivery of medicines to older patients does have complexities, some of which are difficult to overcome, one of the main areas that can be improved within the medication-taking process is to implement support mechanisms to help people to be more adherent. This is notwithstanding the fact that changes in adherence without medication review can lead to problems: patients who have been non-adherent and suddenly begin to take their medications as prescribed may experience adverse effects as a consequence of effectively starting treatment at a higher dose or with more medicines than is necessary. Adherence to suboptimal treatment or harmful therapy is linked to an increased risk of mortality [90]. A medicine review prior to implementation of an adherence 
intervention will help rationalise treatment regimens, remove any inappropriate medicines and ensure the most appropriate dosage form is used.

Overall, there is uncertainty as to the effectiveness of interventions designed to improve medicines use and improve adherence in older people. Equally, there is a relative lack of studies that specifically examine the effects of polypharmacy and multimorbidity on adherence; this is a clear future research priority given these are the issues faced by an older patient population [91]. At present, the literature is dominated by intervention studies that focus on single disease states in specific patient groups, which employ relatively simple interventions, such as reminders to take medicines [92]. To further compound matters, medicines adherence is often arbitrarily split into 'intentional' and 'unintentional' non-adherence within the literature. As a consequence, many of the interventions discussed in the literature are designed to target unintentional adherence and remind patients to take their medication. Another approach that has been employed is the use of reminder packaging as a way of managing patients with polypharmacy in an attempt to reduce unintentional non-adherence. The evidence to support this intervention is inconclusive; marginal improvements have been observed in adherence, although there is limited information on how this approach improves health outcomes [93]. More complex interventions around adherence do show some positive results and are of benefit in long-term care. These interventions are combinations of organisational support, such as improving the convenience of care, electronic reminders, such as sending text messages, smart packaging or telehealth interventions and input from multidisciplinary teams, often involving pharmacist-led initiatives. Despite the improvements in adherence seen with such interventions, there is limited evidence to suggest that a significant clinical benefit can be observed: where outcomes have been measured, they tend to be specific clinical measures, such as blood pressure or INR, rather than end-point outcomes, such as mortality [64]. Use of dose administration aids, which organise doses of tablets and capsules according to when they should be taken, can improve medication management and non-intentional adherence [94]. Dose administration aids may be particularly useful for patients who struggle to manage complex medicine regimen that consists of regularly scheduled medications, or solid oral dose forms that are suitable for packing.

As discussed above, the challenges for older people using inhaled therapies can result in worsening health and increased mortality. Evidence suggests that many people do not receive good education around how to use their inhalers [87] and even when this does happen, the retention of information 
is poor, which results in suboptimal inhaler use within a short period of time [95]. In view of this, Bouwmeetser et al. implemented a pharmacist-led programme to improve inhaler use amongst a community-dwelling group of older patients. The intervention consisted of a baseline test followed by education on how to use inhaler devices then another test to examine improvement in knowledge. Outcomes from this study showed that participants had improved knowledge of inhaler technique, which was sustained over a 6-week period. The participants were also provided with an automatic refill of their prescription for inhalers and thus had sufficient supplies [86]. Similar findings have been observed in other studies; for example, Lindenmeyer et al examined interventions focused on improving adherence to medications in individuals with type 2 diabetes. The results showed an improvement in $\mathrm{HbA} 1 \mathrm{c}$, following a pharmacist-led integrated management and education programme. The authors concluded that although improvement in HBA1c were observed this could not be directly linked to an improvement in adherence [96].

The use of medicines review and deprescribing - defined as 'the process of withdrawal of an inappropriate medication, supervised by a health care professional with the goal of managing polypharmacy and improving outcomes' [97]- for older people is a logical step to address some of the issues raised above. It has been suggested that deprescribing processes have the potential to improve adherence to treatment by educating people about their medicines and making them partners in the decision to take a medicine, irrespective of any net reduction in pill burden. Other proposed benefits of deprescribing on adherence are reduction in financial costs for those individuals who have to pay for their medicines and potential resolution of adverse drug reactions as a consequence of complex polypharmacy. At present, however, there is very little evidence of note to support these claims: most of the literature defines cost savings and reductions in overall medicine use, but is not specifically powered to examine changes in important clinical outcomes, such as hospital admission, morbidity or mortality [98]. Given the evidence that polypharmacy does influence adherence, it would seem to be a plausible hypothesis and one worthy of further investigation in randomised control trials.

\section{Conclusion}

Delivering the right treatment at the right dose to older people is a challenging task. Older people have complex health care needs, which are rarely addressed in drug delivery research to inform the development of medicines suitable for use in this population and to ensure therapeutic outcomes. Drug development cycle needs to be tailored to ensure that new drugs can be safely, effectively, and efficiently delivered to older people. The pharmaceutical manufacturing industry should support 
these efforts by using advanced manufacturing technology to provide affordable, convenient and easy-to-use drug forms. Importantly, it is essential to include older people and caregivers in drug design development process to assess usability, packaging and acceptability of medications, and regulatory institutions should request information about drug delivery and acceptability of formulations for older people.

\section{Funding}

DG is supported by the Australian National Health and Medical Research Dementia Leadership Fellowship.

\section{Conflicts of Interest Statement}

The authors declare that there is no conflict of interest. 


\section{References}

[1]. L.J. Donaldson, E.T. Kelley, N. Dhingra-Kumar, M.P. Kieny, A. Sheikh. Medication Without Harm: WHO's Third Global Patient Safety Challenge. Lancet. 2017;389(10080):1680-1.

[2]. D. Gnjidic, M. Tinetti, H.G. Allore. Assessing medication burden and polypharmacy: finding the perfect measure. Expert review of clinical pharmacology. 2017;10(4):345-7.

[3]. D. Gnjidic, S.N. Hilmer, F. Blyth, V. Naganathan, R.G. Cumming, D. Handelsman, et al. High risk prescribing and incidence of frailty among older community-dwelling men. Clin Pharmacol Ther. 2012;91:521-8.

[4]. E.D. Kantor, C.D. Rehm, J.S. Haas, A.T. Chan, E.L. Giovannucci. Trends in Prescription Drug Use Among Adults in the United States From 1999-2012. JAMA. 2015;314(17):1818-31.

[5]. R.E. Hubbard, N.M. Peel, I.A. Scott, J.H. Martin, A. Smith, P.I. Pillans, et al. Polypharmacy among inpatients aged 70 years or older in Australia. Med J Aust. 2015;202(7):373-7.

[6]. B. Schottker, K.U. Saum, D.C. Muhlack, L.K. Hoppe, B. Holleczek, H. Brenner. Polypharmacy and mortality: new insights from a large cohort of older adults by detection of effect modification by multi-morbidity and comprehensive correction of confounding by indication. Eur J Clin Pharmacol. 2017;73(8):1041-8.

[7]. J. Breitkreutz, J. Boos. Paediatric and geriatric drug delivery. Expert Opin Drug Deliv. 2007;4(1):37-45.

[8]. S. Stegemann. Challenges and opportunities in the design of age-appropriate drug products. Z Gerontol Geriatr. 2012;45(6):479-84.

[9]. European Medicines Agency (EMA). Committee for Medicinal Products for Human Use (CHMP). Concept paper on the need for a reflection paper on quality aspects of medicines for older people. EMA/165974/2013 20 March 2013.

[10]. A.J. McLean, D.G. Le Couteur. Aging biology and geriatric clinical pharmacology. Pharmacol Rev. 2004;56(2):163-84.

[11]. S.R. Barczi, P.A. Sullivan, J. Robbins. How should dysphagia care of older adults differ? Establishing optimal practice patterns. Semin Speech Lang. 2000;21(4):347-61.

[12]. A. Lee, Y.Y. Sitoh, P.K. Lieu, S.Y. Phua, J.J. Chin. Swallowing impairment and feeding dependency in the hospitalised elderly. Ann Acad Med Singapore. 1999;28(3):371-6.

[13]. C.M. Steele, C. Greenwood, I. Ens, C. Robertson, R. Seidman-Carlson. Mealtime difficulties in a home for the aged: not just dysphagia. Dysphagia. 1997;12(1):43-50; discussion 1.

[14]. E.T.L. Lau, K.J. Steadman, J.A.Y. Cichero, L.M. Nissen. Dosage form modification and oral drug delivery in older people. Adv Drug Deliv Rev. 2018.

[15]. S. Stegemann, M. Gosch, J. Breitkreutz. Swallowing dysfunction and dysphagia is an unrecognized challenge for oral drug therapy. Int J Pharm. 2012;430(1-2):197-206.

[16]. N. Mercovich, G.J. Kyle, M. Naunton. Safe to crush? A pilot study into solid dosage form modification in aged care. Australas J Ageing. 2014;33(3):180-4.

[17]. M. Caussin, W. Mourier, S. Philippe, C. Capet, M. Adam, N. Reynero, et al. [Crushing drugs in geriatric units: an "handicraft" practice with frequent errors which imposed recommendations]. La Revue de medecine interne / fondee par la Societe nationale francaise de medecine interne. 2012;33(10):546-51.

[18]. M.S. Fischbach, J.L. Gold, M. Lee, J.M. Dergal, G.M. Litner, P.A. Rochon. Pill-splitting in a long-term care facility. Cmaj. 2001;164(6):785-6.

[19]. J.T. Schiele, R. Quinzler, H.D. Klimm, M.G. Pruszydlo, W.E. Haefeli. Difficulties swallowing solid oral dosage forms in a general practice population: prevalence, causes, and relationship to dosage forms. Eur J Clin Pharmacol. 2013;69(4):937-48.

[20]. A.B. Overgaard, J. Hojsted, R. Hansen, J. Moller-Sonnergaard, L.L. Christrup. Patients' evaluation of shape, size and colour of solid dosage forms. Pharm World Sci. 2001;23(5):185-8.

[21]. B. Liu, M.R. Dion, M.M. Jurasic, G. Gibson, J.A. Jones. Xerostomia and salivary hypofunction in vulnerable elders: prevalence and etiology. Oral Surg Oral Med Oral Pathol Oral Radiol.

2012;114(1):52-60. 
[22]. R.O. Dantas, M.A. Nobre Souza. Dysphagia induced by chronic ingestion of benzodiazepine. Am J Gastroenterol. 1997;92(7):1194-6.

[23]. F. Gonzalez. Extrapyramidal syndrome presenting as dysphagia: a case report. Am J Hosp Palliat Care. 2008;25(5):398-400.

[24]. J.T. Stewart. Dysphagia associated with risperidone therapy. Dysphagia. 2003;18(4):274-5. [25]. M. Miarons, L. Campins, E. Palomera, M. Serra-Prat, M. Cabre, L. Rofes. Drugs Related to Oropharyngeal Dysphagia in Older People. Dysphagia. 2016;31(5):697-705.

[26]. S.N. Hilmer, D. Gnjidic. Prescribing for frail older people. Aust Prescr. 2017;40(5):174-8.

[27]. M.J. Dolton, B.D. Roufogalis, A.J. McLachlan. Fruit juices as perpetrators of drug interactions: the role of organic anion-transporting polypeptides. Clin Pharmacol Ther. 2012;92(5):622-30.

[28]. N. Garin, A. Koyanagi, S. Chatterji, S. Tyrovolas, B. Olaya, M. Leonardi, et al. Global Multimorbidity Patterns: A Cross-Sectional, Population-Based, Multi-Country Study. J Gerontol A Biol Sci Med Sci. 2016;71(2):205-14.

[29]. M.E. Salive. Multimorbidity in older adults. Epidemiol Rev. 2013;35:75-83.

[30]. K. Barnett, S.W. Mercer, M. Norbury, G. Watt, S. Wyke, B. Guthrie. Epidemiology of multimorbidity and implications for health care, research, and medical education: a cross-sectional study. Lancet. 2012;380(9836):37-43.

[31]. R. Palladino, J. Tayu Lee, M. Ashworth, M. Triassi, C. Millett. Associations between multimorbidity, healthcare utilisation and health status: evidence from 16 European countries. Age Ageing. 2016;45(3):431-5.

[32]. M.T. Schram, D. Frijters, E.H. van de Lisdonk, J. Ploemacher, A.J. de Craen, M.W. de Waal, et al. Setting and registry characteristics affect the prevalence and nature of multimorbidity in the elderly. J Clin Epidemiol. 2008;61(11):1104-12.

[33]. D. Kinnear, J. Morrison, L. Allan, A. Henderson, E. Smiley, S.A. Cooper. Prevalence of physical conditions and multimorbidity in a cohort of adults with intellectual disabilities with and without Down syndrome: cross-sectional study. BMJ open. 2018;8(2):e018292.

[34]. A. Kingston, L. Robinson, H. Booth, M. Knapp, C. Jagger, M. project. Projections of multimorbidity in the older population in England to 2035: estimates from the Population Ageing and Care Simulation (PACSim) model. Age Ageing. 2018;47(3):374-80.

[35]. B. Shad, A. Ashouri, T. Hasandokht, F. Rajati, A. Salari, M. Naghshbandi, et al. Effect of multimorbidity on quality of life in adult with cardiovascular disease: a cross-sectional study. Health Qual Life Outcomes. 2017;15(1):240.

[36]. P.D. St John, S.L. Tyas, V. Menec, R. Tate. Multimorbidity, disability, and mortality in community-dwelling older adults. Can Fam Physician. 2014;60(5):e272-80.

[37]. L. Mondor, C.J. Maxwell, D.B. Hogan, S.E. Bronskill, A. Gruneir, N.E. Lane, et al. Multimorbidity and healthcare utilization among home care clients with dementia in Ontario, Canada: A retrospective analysis of a population-based cohort. PLoS Med. 2017;14(3):e1002249.

[38]. A. Calderon-Larranaga, B. Poblador-Plou, F. Gonzalez-Rubio, L.A. Gimeno-Feliu, J.M. AbadDiez, A. Prados-Torres. Multimorbidity, polypharmacy, referrals, and adverse drug events: are we doing things well? Br J Gen Pract. 2012;62(605):e821-6.

[39]. S.V. Doubova Dubova, H. Reyes-Morales, P. Torres-Arreola Ldel, M. Suarez-Ortega. Potential drug-drug and drug-disease interactions in prescriptions for ambulatory patients over 50 years of age in family medicine clinics in Mexico City. BMC Health Serv Res. 2007;7:147.

[40]. C.I. Lindblad, J.T. Hanlon, C.R. Gross, R.J. Sloane, C.F. Pieper, E.R. Hajjar, et al. Clinically important drug-disease interactions and their prevalence in older adults. Clin Ther. 2006;28(8):113343.

[41]. C.I. Lindblad, M.B. Artz, C.F. Pieper, R.J. Sloane, E.R. Hajjar, C.M. Ruby, et al. Potential drugdisease interactions in frail, hospitalized elderly veterans. Ann Pharmacother. 2005;39(3):412-7.

[42]. A.G. Johnson, T.V. Nguyen, R.O. Day. Do nonsteroidal anti-inflammatory drugs affect blood pressure? A meta-analysis. Ann Intern Med. 1994;121(4):289-300. 
[43]. G.C. Machado, C.G. Maher, P.H. Ferreira, M.B. Pinheiro, C.W. Lin, R.O. Day, et al. Efficacy and safety of paracetamol for spinal pain and osteoarthritis: systematic review and meta-analysis of randomised placebo controlled trials. BMJ. 2015;350:h1225.

[44]. S. Derry, P. Conaghan, J.A. Da Silva, P.J. Wiffen, R.A. Moore. Topical NSAIDs for chronic musculoskeletal pain in adults. Cochrane Database Syst Rev. 2016;4:CD007400.

[45]. Osteoarthritis: care and management. Clinical Guideline [CG177] 2014.

https://www.nice.org.uk/guidance/cg177.

[46]. D. Carnes, Y. Anwer, M. Underwood, G. Harding, S. Parsons, T.s. team. Influences on older people's decision making regarding choice of topical or oral NSAIDs for knee pain: qualitative study. BMJ. 2008;336(7636):142-5.

[47]. Gavison Advance Summary of Product Characteristics.

https://www.medicines.org.uk/emc/product/6187/smpc.

[48]. Paracetamol Effervescent 500 mg Tablets Summary of Product Characteristics.

https://www.medicines.org.uk/emc/product/2123/smpc.

[49]. Sodium intake for adults and children. World Health Organisation. 2012.

http://www.who.int/nutrition/publications/guidelines/sodium intake printversion.pdf.

[50]. Salt Reduction Targets for 2017. Public Health England. March

2017.https://assets.publishing.service.gov.uk/government/uploads/system/uploads/attachment da ta/file/604338/Salt reduction targets for 2017.pdf.

[51]. L. Douglas, M. Akil. Sodium in soluble paracetamol may be linked to raised blood pressure. BMJ. 2006;332(7550):1133.

[52]. K. Siau, A. Khanna. Hypernatremia secondary to soluble paracetamol use in an elderly man: a case report. Cases J. 2009;2:6707.

[53]. J. George, W. Majeed, I.S. Mackenzie, T.M. Macdonald, L. Wei. Association between cardiovascular events and sodium-containing effervescent, dispersible, and soluble drugs: nested case-control study. BMJ. 2013;347:f6954.

[54]. Y. Kafaei Shirmanesh, M.D. Jones. Physical ability of people with rheumatoid arthritis and age-sex matched controls to use four commonly prescribed inhaler devices. Respiratory medicine. 2018;135:12-4.

[55]. R.A. Elliott, J.L. Marriott. Standardised assessment of patients' capacity to manage medications: a systematic review of published instruments. BMC Geriatr. 2009;9:27.

[56]. Committee on Identifying and Preventing Medication Errors. Preventing medication errors: quality chasm series. Washington DC: The National Academies Press, 2007.

[57]. Look-alike Sound-alike (LA/SA) Health Product Names. https://www.canada.ca/en/healthcanada/services/drugs-health-products/biologics-radiopharmaceuticals-genetic-therapies/look-

alike-sound-alike/issue-analysis-summary-look-alike-sound-alike-health-product-names-

development-comprehensive-policy-recommendation-look-alike-sound-alike.html.

[58]. Cohen MR. Chapter 7. The role of drug packaging and labelling in medication errors. In Medication Errors, 2nd Edition. 2007.

[59]. Name Differentiation Project. US Food \& Drug Administration:

https://www.fda.gov/drugs/drugsafety/medicationerrors/ucm164587.htm.

[60]. B.L. Lambert, S.R. Schroeder, W.L. Galanter. Does Tall Man lettering prevent drug name confusion errors? Incomplete and conflicting evidence suggest need for definitive study. BMJ Qual Saf. 2016;25(4):213-7.

[61]. MHRA is working with industry to make the packing of medicines used in Alzheimer's disease more user-friendly: https://www.gov.uk/government/news/mhra-is-working-with-industry-to-makethe-packaging-of-medicines-used-in-alzheimers-disease-more-user-friendly].

[62]. NSW Health. Safety Notice 004/08. 05 February 2008.

http://www.health.nsw.gov.au/sabs/Documents/2008-sn-004.pdf.

[63]. M.R. DiMatteo, P.J. Giordani, H.S. Lepper, T.W. Croghan. Patient adherence and medical treatment outcomes: a meta-analysis. Med Care. 2002;40(9):794-811. 
[64]. R.B. Haynes, E. Ackloo, N. Sahota, H.P. McDonald, X. Yao. Interventions for enhancing medication adherence. Cochrane Database Syst Rev. 2008(2):CD000011.

[65]. N. Britten, F. Stevenson, J. Gafaranga, C. Barry, C. Bradley. The expression of aversion to medicines in general practice consultations. Soc Sci Med. 2004;59(7):1495-503.

[66]. P.J. O'Connor. Improving medication adherence: challenges for physicians, payers, and policy makers. Arch Intern Med. 2006;166(17):1802-4.

[67]. Banik S, Ray D. Analysing the pattern of prescription non-compliance in patients of a cardiac and diabetic clinic of AGMC \& GBP hospital. Australasian Medical Journal. 2012;5(1):61).

[68]. D.M. Mann, M. Woodward, P. Muntner, L. Falzon, I. Kronish. Predictors of nonadherence to statins: a systematic review and meta-analysis. Ann Pharmacother. 2010;44(9):1410-21.

[69]. S.J. Rolnick, P.A. Pawloski, B.D. Hedblom, S.E. Asche, R.J. Bruzek. Patient characteristics associated with medication adherence. Clin Med Res. 2013;11(2):54-65.

[70]. W.F. Gellad, J.L. Grenard, Z.A. Marcum. A systematic review of barriers to medication adherence in the elderly: looking beyond cost and regimen complexity. Am J Geriatr Pharmacother. 2011;9(1):11-23.

[71]. V.S. Conn, A.R. Hafdahl, P.S. Cooper, T.M. Ruppar, D.R. Mehr, C.L. Russell. Interventions to improve medication adherence among older adults: meta-analysis of adherence outcomes among randomized controlled trials. Gerontologist. 2009;49(4):447-62.

[72]. F. Smith, S.A. Francis, N. Gray, M. Denham, J. Graffy. A multi-centre survey among informal carers who manage medication for older care recipients: problems experienced and development of services. Health \& social care in the community. 2003;11(2):138-45.

[73]. V. Selak, C.R. Elley, C. Bullen, S. Crengle, A. Wadham, N. Rafter, et al. Effect of fixed dose combination treatment on adherence and risk factor control among patients at high risk of cardiovascular disease: randomised controlled trial in primary care. BMJ. 2014;348:g3318.

[74]. B.C. Wimmer, A.J. Cross, N. Jokanovic, M.D. Wiese, J. George, K. Johnell, et al. Clinical Outcomes Associated with Medication Regimen Complexity in Older People: A Systematic Review. J Am Geriatr Soc. 2017;65(4):747-53.

[75]. S. Bangalore, G. Kamalakkannan, S. Parkar, F.H. Messerli. Fixed-dose combinations improve medication compliance: a meta-analysis. Am J Med. 2007;120(8):713-9.

[76]. E.Y. Chen, J.K. Sluggett, J. Ilomaki, S.N. Hilmer, M. Corlis, L.J. Picton, et al. Development and validation of the Medication Regimen Simplification Guide for Residential Aged CarE (MRS GRACE). Clin Interv Aging. 2018;13:975-86.

[77]. P. Kozarewicz. Regulatory perspectives on acceptability testing of dosage forms in children. Int J Pharm. 2014;469(2):245-8.

[78]. C. Haw, J. Stubbs, G. Dickens. An observational study of medication administration errors in old-age psychiatric inpatients. Int J Qual Health Care. 2007;19(4):210-6.

[79]. J. Marquis, M.P. Schneider, V. Payot, A.C. Cordonier, O. Bugnon, K.E. Hersberger, et al. Swallowing difficulties with oral drugs among polypharmacy patients attending community pharmacies. Int J Clin Pharm. 2013;35(6):1130-6.

[80]. S. Logrippo, G. Ricci, M. Sestili, M. Cespi, L. Ferrara, G.F. Palmieri, et al. Oral drug therapy in elderly with dysphagia: between a rock and a hard place! Clin Interv Aging. 2017;12:241-51.

[81]. D.M. Suiter, S.B. Leder. Clinical utility of the 3-ounce water swallow test. Dysphagia. 2008;23(3):244-50.

[82]. Y. Miura, Y. Morita, H. Koizumi, T. Shingai. Effects of taste solutions, carbonation, and cold stimulus on the power frequency content of swallowing submental surface electromyography. Chemical senses. 2009;34(4):325-31.

[83]. A. Gupta, N. Chidambaram, M.A. Khan. An index for evaluating difficulty of Chewing Index for chewable tablets. Drug Dev Ind Pharm. 2015;41(2):239-43.

[84]. G. Carnaby-Mann, M. Crary. Pill swallowing by adults with dysphagia. Archives of otolaryngology--head \& neck surgery. 2005;131(11):970-5. 
[85]. A.S. Melani, D. Zanchetta, N. Barbato, P. Sestini, C. Cinti, P.A. Canessa, et al. Inhalation technique and variables associated with misuse of conventional metered-dose inhalers and newer dry powder inhalers in experienced adults. Ann Allergy Asthma Immunol. 2004;93(5):439-46.

[86]. C. Bouwmeester, J. Kraft, K.M. Bungay. Optimizing inhaler use by pharmacist-provided education to community-dwelling elderly. Respiratory medicine. 2015;109(10):1363-8.

[87]. A.S. Melani, M. Bonavia, V. Cilenti, C. Cinti, M. Lodi, P. Martucci, et al. Inhaler mishandling remains common in real life and is associated with reduced disease control. Respiratory medicine. 2011;105(6):930-8.

[88]. A. Roy, K. Battle, L. Lurslurchachai, E.A. Halm, J.P. Wisnivesky. Inhaler device, administration technique, and adherence to inhaled corticosteroids in patients with asthma. Prim Care Respir J. 2011;20(2):148-54.

[89]. S.P. Newman, W.W. Busse. Evolution of dry powder inhaler design, formulation, and performance. Respiratory medicine. 2002;96(5):293-304.

[90]. S.H. Simpson, D.T. Eurich, S.R. Majumdar, R.S. Padwal, R.T. Tsuyuki, J. Varney, et al. A metaanalysis of the association between adherence to drug therapy and mortality. BMJ.

2006;333(7557):15.

[91]. R. Ryan, N. Santesso, D. Lowe, S. Hill, J. Grimshaw, M. Prictor, et al. Interventions to improve safe and effective medicines use by consumers: an overview of systematic reviews. Cochrane Database Syst Rev. 2014(4):CD007768.

[92]. S.M. Smith, E. Wallace, T. O'Dowd, M. Fortin. Interventions for improving outcomes in patients with multimorbidity in primary care and community settings. Cochrane Database Syst Rev. 2016;3:CD006560.

[93]. C.J. Heneghan, P. Glasziou, R. Perera. Reminder packaging for improving adherence to selfadministered long-term medications. Cochrane Database Syst Rev. 2006(1):CD005025.

[94]. R.A. Elliot. Appropriate use of dose administration aids. Aust Prescr. 2014;37.

[95]. C.J. Nimmo, D.N. Chen, S.M. Martinusen, T.L. Ustad, D.N. Ostrow. Assessment of patient acceptance and inhalation technique of a pressurized aerosol inhaler and two breath-actuated devices. Ann Pharmacother. 1993;27(7-8):922-7.

[96]. A. Lindenmeyer, H. Hearnshaw, E. Vermeire, P. Van Royen, J. Wens, Y. Biot. Interventions to improve adherence to medication in people with type 2 diabetes mellitus: a review of the literature on the role of pharmacists. J Clin Pharm Ther. 2006;31(5):409-19.

[97]. E. Reeve, D. Gnjidic, J. Long, S. Hilmer. A systematic review of the emerging de fi nition of 'deprescribing' with network analysis: implications for future research and clinical practice. $\mathrm{Br} \mathrm{J}$ Clin Pharmacol. 2015;80(6):1254-68.

[98]. D. Gnjidic, D.G. Le Couteur, L. Kouladjian, S.N. Hilmer. Deprescribing Trials: Methods to reduce polypharmacy and the impact on prescribing and clinical outcomes. Clin Geriatr Med. 2012;28:237-53. 